\title{
TIDAL VOLUME DEPENDENCE ON THE VENTILATORY FREQUENCY AND ALVEOLAR COMPLIANCE IN HFOV
}

\author{
Rožánek M, Horáková Z, Padertová B, Ráfl J, Roubík K \\ Faculty of biomedical engineering, Czech Technical University in Prague, Czech Republic
}

\author{
rozanek@fbmi.cvut.cz
}

\begin{abstract}
High frequency oscillatory ventilation has limited possibility of monitoring of intrapulmonary parameters. We have measured tidal volume during high frequency oscillatory ventilation by external monitor. The experimental circuit consisted of ventilator 3100 with its patient circuit, lung simulator 5600i and monitor Florian for measuring of tidal volume. Tidal volume was measured for different values of alveolar compliance that were set on the lung simulator. Measurement was repeated for ventilatory frequencies 3, 5, 7 and $10 \mathrm{~Hz}$. Ventilatory parameters were held constant during the experiment. We have found that change of alveolar compliance has minimal effect on the delivered tidal volume.
\end{abstract}

Keywords: High frequency oscillatory ventilation, alveolar compliance, tidal volume

\section{Introduction}

Artificial lung ventilation can be a cause of ventilator induced lung injury because of barotrauma or overdistension. Present regimens of artificial lung ventilation use inversed pressure compared with spontaneous breathing where a negative pressure causes the inspirium. The modern trend is to minimize the ventilatory pressures and therefore protective ventilatory regimens are introduced.

High frequency oscillatory ventilation (HFOV) is nonconventional regimen of artificial lung ventilation. Frequency 5-15 Hz and small tidal volumes $1-2 \mathrm{~mL} / \mathrm{kg}$ are used during HFOV. HFOV theoretically meets the strategy of protective ventilatory regimen.

It was shown that normocapnic HFOV affects differently pulmonary and extrapulmonary form of ARDS [1]. These forms of ARDS differ in mechanical properties of the respiratory system $[2,3]$.

The results from simulations that were conducted on the mathematical model of the respiratory system show that airway resistance substantially affects the impedance of the respiratory system and tidal volume whereas the effect of the alveolar compliance is very small in HFOV [4]. HFOV is used mainly as a rescue therapy. It is not still clear what type of patients has a benefit from the use of HFOV although it has been permanently studied. It is generally accepted that HFOV should not be used in patients with diseases like chronic obstructive pulmonary disease that are characterized mainly by obstruction of the airways or asthma $[5,6]$.

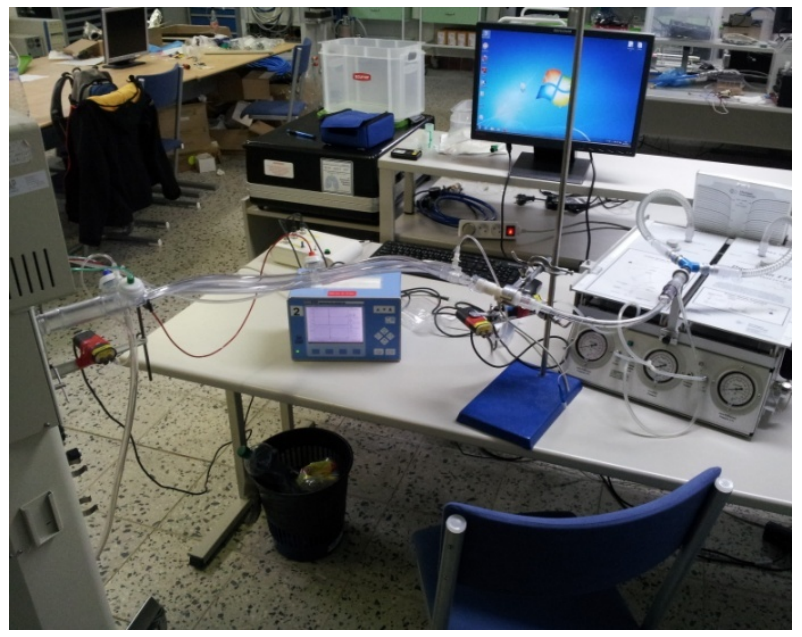

Figure 1: Complete ventilator circuit with high frequency oscillatory ventilator 3100 , monitor Florian and lung simulator 5600i.

Aim of the study is to evaluate by an experimental measurement dependence of tidal volume during HFOV on the alveolar compliance for different ventilatory frequencies.

\section{Methods}

We used ventilator 3100 (Sensormedics, USA) as a high frequency oscillatory ventilator. Monitor Florian (Acutronic Medical Systems, Switzerland) was used for measuring of tidal volume $V_{t}$ in the ventilatory circuit. Respiratory system was modelled by lung simulator $5600 \mathrm{i}$ (Michigan Instruments, USA). Experimental setting is depicted in Fig. 1.

Two resistors Rp5 included in the model were used to simulate the airway resistance during whole experiment. Mean airway pressure (MAP) was maintained at $12 \mathrm{cmH}_{2} \mathrm{O}$, inspirium/expirium ratio was $1: 1$ and amplitude of pressure oscillations $\Delta \mathrm{P}$ was $40 \mathrm{cmH}_{2} \mathrm{O}$. These parameters were held constant during whole experiment.

Different frequencies were set up on the ventilator during the measurement 3,5, 7 and $10 \mathrm{~Hz}$. Compliance was differing across the frequencies: $0.1,0.075,0.05,0.025$ and $0.01 \mathrm{~L} / \mathrm{cmH}_{2} \mathrm{O}$. Endotracheal tube with internal diameter $9 \mathrm{~mm}$ was placed between the ventilator and the simulator. 


\section{Results}

Values of tidal volume are summarized in Tab. 1 for different ventilatory frequencies corresponding to HFOV and for different values of alveolar compliance.

Table 1: Tidal volume and pressure amplitude for different ventilatory frequencies and different levels of alveolar compliance.

\begin{tabular}{|c|c|c|}
\hline $\begin{array}{c}\text { Frequency } \\
(\mathrm{Hz})\end{array}$ & $\begin{array}{l}\text { Compliance } \\
\left(\mathrm{L} / \mathrm{cmH}_{2} \mathrm{O}\right)\end{array}$ & $\begin{array}{c}\text { Tidal volume } \\
\mathrm{V}_{\mathrm{t}}(\mathrm{mL})\end{array}$ \\
\hline \multirow[t]{5}{*}{$x^{2}$} & 0.1 & 145 \\
\hline & 0.075 & 144 \\
\hline & 0.050 & 144 \\
\hline & 0.025 & 145 \\
\hline & 0.010 & 145 \\
\hline \multirow{5}{*}{5} & 0.1 & 95 \\
\hline & 0.075 & 94 \\
\hline & 0.050 & 94 \\
\hline & 0.025 & 95 \\
\hline & 0.010 & 99 \\
\hline \multirow{5}{*}{7} & 0.1 & 71 \\
\hline & 0.075 & 72 \\
\hline & 0.050 & 73 \\
\hline & 0.025 & 73 \\
\hline & 0.010 & 74 \\
\hline \multirow{5}{*}{10} & 0.1 & 45 \\
\hline & 0.075 & 46 \\
\hline & 0.050 & 46 \\
\hline & 0.025 & 47 \\
\hline & 0.010 & 49 \\
\hline
\end{tabular}

\section{Discussion}

The results of the conducted experiment suggest that change of the alveolar compliance has very small effect upon the delivered tidal volume during all frequencies corresponding to HFOV usage. Minimum effect was observed at all ventilatory frequencies $3,5,7$ and $10 \mathrm{~Hz}$.

The results are in concordance with the simulations conducted on the mathematical model that was designed according to the anatomy of the lungs [4]. It was shown that compliance of alveoli has only slight effect upon the impedance of the respiratory system. The effect of alveolar compliance upon the impedance of the respiratory system is depicted in Fig. 2. It means that alveolar compliance has also small effect upon the tidal volume at pressure-controlled regimens of artificial lung ventilation at frequencies that correspond to use of HFOV.

Some studies show that patients with severe ARDS have benefit from use of HFOV [7]. These patients have decreased alveolar compliance in many cases and HFOV seems to be able to ventilate adequately lung with similar settings as for the healthy lung.

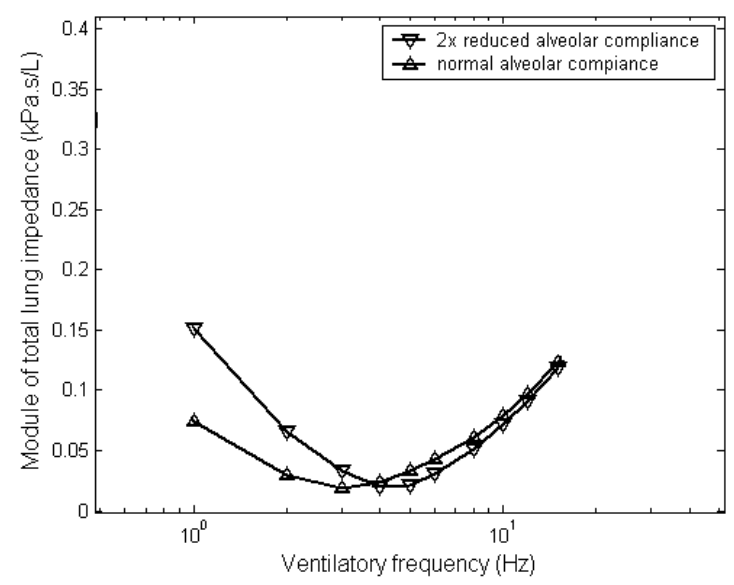

Figure 2: Dependence of lung impedance on the ventilatory frequency for HFOV simulated for normal and decreased alveolar compliance.

\section{Acknowledgement}

This research has been supported by grant SGS11/171/OHK4/3T/17.

\section{Bibliography}

[1] Pachl J, Roubík K, Waldauf P, Fric M, Zábrodský V.: Normocapnic High-frequency oscillatory ventilation affects differently extrapulmonary and pulmonary forms of acute respiratory distress syndrome in adults, Physiological Research, vol. 55, pp. 15-24, 2006.

[2] Gattinoni L, Pelosi P, Suter PM, et al.: Acute respiratory distress syndrome caused by pulmonary and extrapulmonary disease. Different syndromes?, Am J Respir Crit Care Med, vol. 158, 99. 3-11, 1998.

[3] Pelosi P, D'Onofrio D, Chiumello D, et al.: Pulmonary and extrapulmonary acute respiratory distress syndrome are different, Eur Respir J, vol. 22, pp. 48s-56s, 2003.

[4] Rozanek M, Roubík K, Horáková Z.: Simulation of the Different Respiratory Mechanics Effect upon the Efficiency of Artificial Lung Ventilation Using Mathematical Model of the Respiratory System, in: World Congress on Medical Physics and Biomedical Engineering, Munich, Germany, pp. 1674-1676, 2009, ISBN 978-3-642-03881-5.

[5] Chan KPW, Stewart TE, Mehta S.: High-frequency oscillatory ventilation for adult patients with ARDS, Chest, vol. 131, pp. 1907-1916, 2007.

[6] Fessler HE, Derdak S, Ferguson ND, et al.: A protocol for high-frequency oscillatory ventilation in adults: Results from a roundtable discussion. Crit Care Med, vol. 35, pp. 1649-1654, 2007.

[7] Sud S, Sud M, Friedrich JO, Meade MO, Ferguson ND, Wunsch H, Adhikari NK.: High frequency oscillation in patients with acute lung injury and acute respiratory distress syndrome (ARDS): systematic review and metaanalysis, $B M J$, vol. 18, p. c2327, May 2010. 